\title{
Diagnostic medical ultrasound awareness among women in Cameroon
}

\begin{abstract}
Ultrasonography (also known as Ultrasound) is vital for the health care of women. Most women in Cameroon are still ignorant of the value of this tool, especially those in rural areas. Many factors such as level of education, cost of purchasing and training Sonographers, low level of development, government policies, training of medical health care workers, insufficient infrastructures are all seen to influence the knowledge of Cameroon women on Ultrasound (US). This knowledge gap motivated the writing of this opinion paper whose main aim is to document on the ultrasound awareness among Cameroon women, with hopes that its outcome would positively influence policies in Cameroon, regarding the health care of women.
\end{abstract}

Keywords: Ultrasound; Ultrasonography; Medical Imaging; Radiations; Testicular, Thyroid, Abdominopelvic, Doppler, Cardiac
Volume 4 Issue 4 - 2017

\author{
Samuel Nambile Cumber,' Keneth Nkeh \\ Nchanji⿱ \\ 'Department of Nursing \& Public Health, University of Kwazulu- \\ Natal, South Africa \\ ${ }^{2}$ St Louis University Institute of health and Biomedical Science \\ Bamenda, Cameroon
}

\begin{abstract}
Correspondence: Samuel Nambile Cumber, Discipline of Public Health Medicine, Department of Nursing \& Public Health, College of Health Sciences, University of Kwazulu-Natal, Durban, South Africa, Email samuelcumber@yahoo.com
\end{abstract}

Received: September 20, 2017 | Published: November 02,

2017

\section{Introduction}

Medical Imaging is indispensable for successful medical practice. It embodies various imaging modalities, with Ultrasonography also known as Ultrasound being one of them. The use of Ultrasound (US) was introduced in the 1950s for medical diagnosis and there has been tremendous increase in the technology of this imaging tool. Ultrasonography is a medical diagnostic imaging modality, which uses sound waves of frequency greater than 20 Kilohertz $(20 \mathrm{KHz})$. Sound waves are non-ionizing radiations unlike X- Rays, gamma rays, alpha particles, or neutrons. Ultrasound is being generated with the help of the ultrasound machine, using the piezo-electric principle.

The medical applications of ultrasound are varied, ranging from Obstetric and Gynecological ultrasound scans, breast ultrasound in women to testicular, thyroid, abdominopelvic, Doppler, cardiac and musculoskeletal ultrasound; diagnosing a wide variety of diseases such as cystic lesions, fibroids, masses, abscesses collection, to say the least. US use is generally up till date considered to be safe and has tremendous benefits to women considering their physiology and also radiation effects related issues. ${ }^{1,2}$

Ultrasound use in women is dominant in the areas of Obstetrics and Gynecology, breast imaging, and also musculoskeletal, thoracic and abdominal imaging. Women can do an ultrasound scan to diagnose lumps in the breast, to evaluate pregnancy status (obstetrics), to evaluate the pelvic organs and the related structures in the pelvis (gynecology). ${ }^{2-6}$ The benefits and rational for the wide advocate of ultrasound use in women are because ultrasound use is relatively cheap and easily affordable and accessible, Ultrasound scans are done in real time, there is no use of ionizing radiations such as X-Rays with side effects, sectional images obtained give detailed information about the structures scanned, requires minimal patient preparations such as a filled urinary bladder for pelvic US and will also effectively diagnose soft tissue problems better than two dimensional plain radiography. ${ }^{1}$

\section{Current level of diagnostic medical ultrasou-} nd awareness among cameroon women

Despite these numerous benefits of ultrasound in women, studies reveal that Ultrasound awareness among women in Cameroon still remains low. ${ }^{6}$ This ignorance among women cuts across many African countries. Such ignorance has associated with them potential consequences. ${ }^{6}$ Based on a study conducted by Joshua et al.${ }^{6}$ out of a total of 200 women who were interviewed, 33 respondents $(16.8 \%)$ on a total of 196 declared that they have never done an US scan, 19(58.4\%) on a total of 190 confessed having received no information on what Ultrasound is all about. About 34 women considered Ultrasound to be unsafe as they believe that, ultrasound uses X-rays which can cause cancer. This really revealed that most women in Cameroon are really ignorant of the use of ultrasound. It is equally worth noting that in this study, most women were within the age group of 25-29years.

Furthermore, based on the World Bank reports of 2014, concerning Cameroon, approximately $10 \%$ of youths in Cameroon lack formal education. ${ }^{7}$ A total of $30 \%$ youths between the ages of $15-24$ years have not completed primary education and only $1 \%$ has completed secondary school, meanwhile $55 \%$ are secondary school dropouts. ${ }^{7}$ Education is vital because it exposes people to knowledge which can influence the view women have about healthcare. This gross ignorance has contributed to many complications in pregnancies such as still births, fetal malformations, infertility due to fibroids in women's uterus, breast cancer; all clinical conditions that can be effectively and safely evaluated on Ultrasound. Besides these consequences are broken relationships, frustrations and unnecessary operations in these women and in some cases death.

Factors contributing to the ignorance of the value of diagnostic medical ultrasound among cameroon women

The level of literacy in relatively low. ${ }^{7}$ Despite the increase in antenatal care uptake in Cameroon, the wide application of ultrasound among women especially expectant women is low. ${ }^{8-10}$ Most antenatal care services do not emphasize on this in Cameroon. ${ }^{9}$ Only few clinics in Cameroon do insist on ultrasound scanning. ${ }^{9}$ The villages and generally rural areas are not also covered. ${ }^{4,8,9}$ Women in these areas are not schooled on ultrasound benefits. Obstetricians and Gynecologists who request most ultrasound exams, do not properly educate these 
women and of the most Nurses and Midwives who frequently interact with women are equally ignorant of the value of ultrasound. ${ }^{4,9}$ This may be due to the nature and design of most of their educational curricula which do not include courses in Obstetrics and Gynaecological Ultrasound, and the antenatal care services being offered to women in Cameroon hardly lay emphasis on the value of ultrasound use unlike in other African countries such as Sierra Leone. ${ }^{11}$ Moreover, most government programs concerning women hardly emphasize the value of ultrasound use which may partly be due to negligence especially on medical imaging training in the country, and as such graduates from this field are not always given first place as regard recruitment and sensitization programs in this country. ${ }^{4-6,12}$ In addition, despite enormous efforts made by the government to educate her citizens and also fulfill the Millennium Development Goals (MDGs), there are still cultural practices in Cameroon which contribute to such ignorance among women. Most people still see educational training of the girl child as a waste of resources and the females should be reserved for marriage. This point is further solidified by the rationale for the Boko Haram crisis in the North of Cameroon, as to them, the girl child should not be educated..$^{7-9}$

\section{Recommendations to address this current problem}

To address this issue, the following proposed and solutions are viewed as vital: medical students and Nursing/Midwifery students should be taught Ultrasonography as a major course, women during antenatal visits should be given lectures on Ultrasonography by Sonographers, sensitization programs on popular national and local Televisions (TV) programs such as Hello, Monday Show and Radio programs such as SOS Doctor should focus on the value of ultrasound, educative fly outs and leaflets on ultrasound, dedicated people should be sent to Women's groups such as "Njangis", the government through the Ministry of women empowerment can sponsor peer educators on Ultrasonography training, Sonographers from Medical Imaging Training schools in the Country should be recruited and deployed to health centers so they can better educate women especially those in the villages and remote areas, screening programs for women could be done, during which US scans can be done, and this will serve as a window to educate the women, such programs should also be made sustainable by the government and Non-governmental organizations (NGOs), for every given intervention, regular evaluation of progress should be done to check the level of awareness and through the wide use of the social networks such as Mobile networks like MTN, Nextel, and Orange. Regular messages should be given to the women and the general population on the value of Ultrasound via those channels. Such programs could be co-sponsored by the Ministries of public Health, Women Empowerment and the Family.

\section{Conclusion}

In a nut shell, if the above solutions are implemented most women will be aware of the importance of ultrasound and its benefits from this very useful Medical Diagnostic Imaging Modality and overall health improvement will be achieved.

\section{Acknowledgements}

None.

\section{Conflict of interest}

Author declares that there is no conflict of interest.

\section{References}

1. Kathryn AG. Ultrasound in Obstetrics and Gynaecology: A Practitioner's Guide. Pasadena, California: Davis Publishing Inc; 2014.

2. https://www.cancer.org/

3. Alfred A, Rabih C, Philipe J, et al. Ultrasound in Obstetrics and Gynaecology: A practical Approach. A Global Library of Women's Medicine. 2014.

4. Hofmeyr GJ. Routine Ultrasound Examination in Early Pregnancy: Is it Worthwhile in Low-Income Countries? Ultrasound Obstetrics and Gynecology. 2009;34(4):367-370.

5. Ultrasound Diagnosis of Early Pregnancy Miscarriage. Institute of obstetricians and Gynecologists, Ireland; 2010.

6. Joshua T, Boniface M, Odile FZ, et al. Routine Prenatal Ultrasonography: The Pregnant Women's Expectations and Safety in a Central African Obstetric Population. Open Journal of Obstetrics and Gynaecology. 2013;3(7):573-576.

7. National Education Profile of Cameroon. Cameroon.

8. Reproductive Health at Glance Cameroon. USA: Washington; 2011.

9. Mbuagbaw LC, Gofin R. A New Measurement for Optimal Antenatal Care: Determinants and Outcomes in Cameroon. Matern Child Health J. 2010;15(8):1427-1434.

10. WHO. World Health Statistics. Maternal and Child Health Journal. 2015;15(8):1427-1434.

11. Donnelly John. How did Sierra Leone provide free health care? Lancet. 2011;377(9775):1393-1396.

12. Agnes B. Gynaecology and Obstetrics: Clinical Protocols and Treatment Guidelines. Ministry of Public Health, Kigali Rwanda; 2012. p. $1-192$. 